CP, 2018, Vol.7 - No15, pp. 59/73 ISSN 2014-6752. Girona (Catalunya). Universitat de Girona. SMOLAK LOZANO, Emilia y CASTILLO ESPARCIA, Antonio: Comunicación política y los think tanks. Estrategias de comunicación en las redes sociales Recibido: 16/05/2018 - Aceptado:27/06/2018

\title{
Comunicación política y los think tanks. Estrategias de comunicación en las redes sociales
}

\section{Political communication of think tanks. Strategic com- munication in social networks.}

\author{
Autores: \\ Emilia Smolak Lozano \\ https://orcid.org/0000-0001-8193-8786 \\ Universidad de Málaga \\ Antonio Castillo Esparcia \\ https://orcid.org/0000-0002-9751-8628 \\ Profesor Catedrático Director de Departamento de Comunicación Audiovisual y Publicidad \\ Universidad de Málaga
}

\section{Resumen}

La presente investigación tiene como objetivo examinar y describir el uso de los social media como estrategia de relaciones públicas de los think tanks para tener presencia en los procesos de implementación de políticas públicas. Para ello se van a analizar los think tanks ubicados en procesos de transformación políticos como son los think tanks asiáticos. Los resultados señalan que en su mayoría $(62.5 \%)$ han implementado las estrategias digitales para establecer relaciones con sus stakeholders: políticos, científicos, periodistas y ciudadanos. Estas incluyen las páginas web y las redes sociales. Dado que el grado de implementación de las estrategias digitales puede variar de forma considerable se ha apostado de un modelo general de valoración de la interactividad que permite construir un benchmark del grado de implementación de la comunicación digital por estas organizaciones.

Palabras claves: Think tanks, estrategia, comunicación digital, relaciones públicas 2.0, redes sociales

\section{Abstract}

The present research aims to examine and describe the reality of the use of Social Media as the organisational communication strategy directed to the external publics in the political sphere. Mostly, it is focused on the application of the social networks by the global think tanks in the transforming regions. The majority of the Asian think tanks (62.5\%) has employed the digital strategies to establish the relationships with their stakeholders: politicians, scientists, journalists and citizens. These digital tools include the development of the web pages and social networks. Given that the extent to which these digital strategies are developed can vary considerably, the authors have designed the general model of evaluation of the grade of the interactivity. This allows to construct the benchmark of digital communication use and its extension among the think tanks on the Asian continent.

Palabras claves: Think tanks, strategy, digital communication, public relations 2.0 , Social Media 


\section{Introducción}

Como admite Van Ruler (2015: 187-188), en la era de la información (Castells, 1996) la esfera pública se incrementa de forma muy feroz y vigorosa gracias al desarrollo de Internet. Los Social Media han causado que no existan límites para la expansión de la información. En definitiva, como afirma Auger (2013), se ha cambiado la forma de comunicación tanto de las organizaciones como de los individuos, lo que dado lugar a la remodelación del "mercado de ideas". De este modo, los Social Media y sobre todo las redes sociales no sólo han contribuido con los espacios para la comunicación interpersonal, sino también han creado el nuevo panorama de comunicación política y organizativa democratizada entre la organización y el público haciéndola más pública, pero con la posibilidad de realizarla a nivel interpersonal (Auger, 2013: 2013). Valentini (2015: 172) enfatiza la democratización de la influencia dentro de la sociedad, que consiste en la libre participación y contribución de los actores en el entorno digital, siendo el vehículo de las transformaciones sociales y culturales. El impacto de los Social Media sobre la democracia fue acompañado, además, con el mismo impacto positivo sobre relaciones públicas como herramienta de comunicación en el debate público dentro del panorama democrático, ya que las plataformas de Social Media y las redes sociales han facilitado el acceso de las organizaciones a promover y persuadir sus ideas en el ciberespacio (Auger, 2013: 369; Verheyden, 2017: 9).

Seitel (2002) engloba relaciones públicas de nuestra época en los procesos de la democracia global del siglo XXI y en el auge de Internet y el desarrollo de las TIC. Wilcox, Cameron y Xifra (2012: 561) apuntan a la dimensión tecnológica e internacional de relaciones públicas modernas que surgió por el desarrollo de Internet, lo que facilita una comunicación inmediata, al instante y directa, características emblemáticas de los Social Media. La relación entre el desarrollo de la comunicación 2.0, cambios democráticos, así como el significado y forma de relaciones públicas modernas, se han reflejado en el concepto de relaciones públicas políticas 2.0 de Painter (2015) y de forma más general en la comunicación política del Rey Morató (2007).

EL estudio se basa en el análisis del contenido de los 40 think tanks asiáticos os recogidos en el informe de McGann del año 2015 "Go to Global Think tanks". Los objetivos son analizar las redes sociales, sus contenidos digitales y la web de estos think tanks, a través de una plantilla adaptada de Capriotti et.al. (2016), basándonos en las siguientes variables: uso de herramientas interactivas en los sitios web corporativos, dimensiones de la presencia en página web, configuración de tácticas de Social Media y estrategia digital, el nivel de interactividad de las mismas, capacidad de crear una comunidad, innovación en adopción de las redes sociales, uso y formatos de contenido, así como características específicas de las redes sociales más empleadas: Facebook y Twitter.

\subsection{Los Think tanks como actor político}

Hoy en día, según los datos de Fred Kuntz de 2013(1), existen 5.500 think tanks en todo el mundo. No obstante, como apuntan varios autores, es un fenómeno bastante joven, al cual se ha prestado una atención limitada desde el ámbito académico y que el fenómeno per se se caracteriza por una considerable pluralidad, ambigüedad y complejidad tanto en su manifestación empírica como desde las perspectivas teóricas que pueblan los estudios sobre este fenómeno. "Think tank is an umbrella term that means different things for different people." (Stone, 1996:8). La actividad de "pensar" (think) y una infraestructura (tank) constituyen el rasgo común de es- 
tas organizaciones y una base universal para la conceptualización de este fenómeno: la fábrica de las ideas que después tienen como objetivo influenciar la actividad política.

De este modo, los think tanks se definen a ellos mismos a través de las relaciones entre si y las demás organizaciones a través de dinámicas de aproximación o diferenciación de otros actores. Los think tanks son actores políticos que desarrollan interrelaciones con otros actores a través de las estrategias de comunicación política. En este sentido, reciben propuestas sociales de resolución de cuestiones políticos y aportan su capacidad investigadora en el proceso de discusión y elaboración de políticas públicas. Esta perspectiva funcional-sistémica y contextualizadora define el think tank como "boundary worker" -el agente transfronterizo interrelacionado con los demás actores a través de complejas dinámicas del contexto (Mendizabal, 2012).

Asimismo, basar el estudio de comunicación de los think tanks en países en transición y el concepto de agente que opera entre distintos campos del sistema social, conlleva varias ventajas. De este modo, este marco teórico se puede considerar una ampliación de las perspectivas teóricas que hablan de las comunidades creadas en torno a la investigación o políticas públicas (Abelson, 2001 y 2006) ya que incluyen más actores o agentes, además de las organizaciones, que se puedan considerar competidores, entre ellos: medios de comunicación, organismos estatales o instituciones académicas, comunidades locales y ciudadanos. En definitiva, dicha ampliación no sólo refleja con más exactitud la realidad de los debates contemporáneos en distintos contextos políticos y sociales, así como los procesos estrictamente políticos, sino también facilita los estudios de los mecanismos de la influencia más allá del proceso directo de elaboración de una política final por los gobernantes, al incluir la dimensión social o mediática que ejerce más influencia en los contextos en procesos de transformación.

\subsection{Relaciones públicas políticas en ciberdemocracia y think tanks}

Xifra (2003:200-201) en su definición de los think tanks subraya la faceta política como la fundamental y nos aporta varios elementos dignos de consideración. Primero, que el rol primordial está vinculado a la dimensión política en la cual los think tanks aparecen como actores principalmente políticos. Segundo, que dentro de su rol político se admite el activismo e influencia de estas organizaciones hacia los cambios. Tercero, que esta dimensión y actividad política se realiza principalmente a través de la comunicación. De este modo, podemos considerar el rol social de los think tanks como complementario, particularmente en los contextos transformantes y democracias jóvenes, donde los think tanks van actuar como actores sociales en apoyo al fomento y avance de sociedad civil.

En el ámbito de las ciencias de la comunicación dominan, por un lado, las perspectivas del marketing de ideas como estrategia para combatir la competencia en el mercado de ideas (Abelson ,2001 y 2006; Xifra, 2008, Xifra y Ponsa,2009, Castillo y Smolak-Lozano, 2012; Mendizábal, 2014), y por otro las de relaciones públicas (Xifra, 2003 y 2008; Castillo 2009; Castillo y Smolak-Lozano, 2012; Castillo, Almansa y Smolak-Lozano, 2015; Lalueza y Girona, 2016). Tal como lo admite el informe de los think tanks más prestigioso, Go To Global Thinks Tanks de McGann (2010-2017), la estrategia digital es imprescindible para los think tanks globales (McGann y Johnson, 2005) con el objetivo de desarrollar una estrategia de comunicación digital que permita gestionar las relaciones con sus públicos claves (modelo de boundary agent de Medvetz, 2008). Esta estrategia tiene que cumplir unas características importantes de interacti- 
vidad y uso de contenido adaptado a la realidad digital y herramientas específicas (Selee, 2013).

Montobbio, (2013: 22) resume que como actores políticos los think tanks "son un instrumento de poder blando (...)." del cual núcleo es la comunicación. El rol principal de un think tank descansa sobre la acción comunicativa dentro del ámbito social y político. Se pone en evidencia el valor estratégico de comunicación en los estudios, tanto teóricos como empíricos, de los think tanks desde la perspectiva funcional-sistémica. De este modo, la comunicación política se manifiesta como el gran aliado de estas organizaciones, ya que los think tanks responden a los procesos de globalización y a los cambios tecnológicos en comunicación. Por este motivo es importante hablar de los principios estratégicos de la comunicación de los think tanks sobre todo en la ciberdemocracia (Castells, 2009). De este modo, cabe analizar la comunicación de los think tanks desde el punto de vista de los paradigmas difusionista y a su vez participativo (Herrero, 2009: 108-109) y como propone Xifra (2003:195), desde el punto de vista de los efectos de la comunicación y modelo de dos grados de flujo comunicativo (two-step flow of communication), aunque en la época de la burbuja del Social Media, debemos considerar también otros modelos comunicativos como por ejemplo las relaciones públicas políticas. Evidentemente, Castillo y Trujillo (2010), Castillo y Castillero (2010), Castillo (2009b, 2009c) y Xifra (2003 y 2008) resaltan la importancia de relaciones públicas en la comunicación en su diversidad de acciones. A esta definición debemos añadir dos aspectos fundamentales desde el punto de vista de la sociedad englobada en la Galaxia de Internet (Castells, 2009): la interacción y la relación. De Rey Morató (1996:175-187) analizando la democracia, posmodernidad y el rol de comunicación política define la última en términos de interacción social entre los participantes y en términos relacionales que al fin y al cabo posibilitan el diálogo en el espacio público, permitiendo un flujo de mensajes recíprocos a través de los medios de comunicación.

Relaciones públicas políticas 2.0 desde la perspectiva conversacional que conlleva la idea de relación mutua, es el ámbito donde juega un papel primordial los conceptos de confianza, entendimiento, reflexión (Van Ruler, 2015: 189) y sobre todo la interactividad (Leiner y Quiring, 2008; Capriotti, Carretón y Castillo, 2016).

La relación es el núcleo y protagonista del análisis de relaciones públicas como sistema comunicativo y sus actores (Xifra, 2003: 65; Coombs y Holladay, 2015:690). En definitiva, la retroalimentación basada en la interactividad se convierte en el núcleo de la relación en los entornos digitales. Gershon (2017: 139) define la interactividad como "habilidad de involucrarse en la comunicación bidireccional (...) y reside en las percepciones y experiencias de los participantes directos en los procesos de comunicación" donde la retroalimentación juega el papel clave (Rodríguez, 2002:109; Ponti y Domingo , 2014: 560).

Con este enfoque y para que las ideas tengan peso y significado en las relaciones interactivas online o que las relaciones sirven para los efectos de las ideas transmitidas, así como impacten e influencien realmente, es decir, tengan el poder de moldear la política pública ("ideas that matter"), necesitamos un enfoque estratégico (Selee, 2013), que se convierta en la herramienta clave, sobre todo, en las organizaciones en transición y que funcione en los contextos marcados por dicha transición, ya que facilitan el cambio a través de las formas interactivas proporcionadas gracias a las estrategias digitales Mendizabal (2012)(2) cuyo abanico es muy amplio y diverso. 


\section{Metodología}

Para determinar el nivel de la interactividad y en general la calidad de las relaciones desarrolladas a través de las herramientas digitales aplicadas dentro de las estrategias digitales de comunicación política de los think tanks dentro del marco de las relaciones públicas políticas, hemos analizado a los think tanks de Asia -Pacífico siendo los incluidos dentro del ranking de los mejores think tanks globales (analizados desde 2011 a 2016, según "Global Go To Think Tank Report" de Profesor McGann de la Universidad de Pennsylvania). En total fueron, los 40 de los mejores think tanks globales de Asia -Pacifico, seleccionados según el informe "Global Go To Think Tank Report" de 2013 y actualizado en 2015. A base de estos objetos de estudio hemos analizado 40 páginas web y un total de 15 Redes Sociales por cada think tank (Facebook, Twitter, Flickr, Pinterest, Youtube, Vimeo, Slideshare, Wikipedia, Scribd, Academia.edu, Linkedin, red local y blog).

Se presenta así una metodología mixta en la que se analiza la presencia de los think tanks en las redes sociales (Figura 1).

Fig.1. Metodología de estudio.

\begin{tabular}{|l|l|l|}
\hline Metodología & Métodos & Técnicas \\
\hline Cuantitativos & $\begin{array}{l}\text { Análisis del contenido } \\
\text { Estudio de opinión } \\
\text { Analítica Web 2.0 }\end{array}$ & $\begin{array}{l}\text { Ficha de análisis /Escala Likert } \\
\text { Encuesta online } \\
\text { Online data mining }\end{array}$ \\
\hline Cualitativos & $\begin{array}{l}\text { Observación } \\
\text { Análisis documental } \\
\text { Análisis textual } \\
\text { Estudio de caso }\end{array}$ & $\begin{array}{l}\text { Observación directa no participativa } \\
\text { Análisis de fuentes secundarios } \\
\text { Redes sociales y su contenido (mensajes) }\end{array}$ \\
\hline
\end{tabular}

Fuente: elaboración propia.

Para ello se ha elaborado una tabla en la que se presenta las herramientas de medición de redes sociales empleadas que nos permitieron a recoger los datos: Fanpage Karma, Likelyzer, Simply Measured, Twitonomy, Tweetreach, Sotrender, HowSociable y SocialMention.

Las variables del estudio fueron las siguientes: uso de a herramientas interactivas y nivel de interactividad en la página web, así como en cuanto a las redes sociales las piezas de contenido, alcance, impresiones, influencia, opinión, tácticas aplicadas y Social Media.

El objetivo general del estudio se enfocaba en determinar cómo se emplean las estrategias digitales de las relaciones públicas políticas de los think tanks globalmente. Para ello, hemos enfocado el análisis en los siguientes objetivos específicos

1. Determinar el nivel de la interactividad de las herramientas digitales de los think tanks.

2. Identificar los modelos de aplicación de las relaciones públicas 2.0 en comunicación política de los think tanks. 
El estudio por tanto se basa en las siguientes hipótesis:

- H1: La implementación de Social Media presenta la diversificación de las estrategias y tácticas de las relaciones públicas políticas 2.0, así como el uso extenso de las plataformas de las redes sociales.

- H2: Los think tanks en su mayoría cuentan con un alto grado de influencia e involucración y sobre todo la interactividad con sus públicos a través de sus redes sociales de presencia.

\section{Resultados}

En general, los think tanks analizados muestran un nivel similar de interactividad a través de las redes de medios sociales, los sitios web y también en términos de adaptación a la nueva realidad 2.0, estando registrados en los niveles inferiores- por debajo y poco más de uno. Han adoptado herramientas, tácticas y estrategias similares respecto a las herramientas y redes sociales de forma similar.

Uso de herramientas interactivas en los sitios web corporativos se presenta de la forma siguiente:

\section{Cuadro 1. Uso de herramientas interactivas en los sitios web corporativos.}

\begin{tabular}{|c|c|c|c|c|c|}
\hline & $\mathrm{N}$ & $\%$ & VA & $\begin{array}{l}\text { Points } \\
\text { Awarded }\end{array}$ & $\begin{array}{l}\text { Medium } \\
\text { Interactivity }\end{array}$ \\
\hline Presenting/Exposure & & & 1 & & \\
\hline Newsletter & 7 & $16 \%$ & & & \\
\hline Intranet & 2 & $4 \%$ & & & \\
\hline Library/Archive/edata/most read & 9 & $19 \%$ & & & \\
\hline gallery & 2 & $4 \%$ & & & \\
\hline $\begin{array}{l}\text { networks/strategic alliances/international } \\
\text { cooperation/partners }\end{array}$ & 5 & $11 \%$ & & & \\
\hline Podcast & 4 & $9 \%$ & & & \\
\hline webinars & 2 & $4 \%$ & & & \\
\hline Media Center/newsroom & 15 & $33 \%$ & & & \\
\hline \multirow[t]{2}{*}{ total: } & 46 & $100 \%$ & & 46 & 0,19 \\
\hline & $17 \%$ & & & & \\
\hline Connecting & & & 2 & & \\
\hline RSS & 10 & $13 \%$ & & & \\
\hline Register/account & 3 & $4 \%$ & & & \\
\hline Contact form & 18 & $23 \%$ & & & \\
\hline Email & 26 & $34 \%$ & & & \\
\hline Links to other centers & 9 & $12 \%$ & & & \\
\hline $\begin{array}{l}\text { Suscription to journals and other } \\
\text { publications }\end{array}$ & 11 & $14 \%$ & & & \\
\hline
\end{tabular}




\begin{tabular}{|c|c|c|c|c|}
\hline total: & 77 & $100 \%$ & 154 & 0,65 \\
\hline \multicolumn{5}{|c|}{$29 \%$} \\
\hline Sharing & & & 3 & \\
\hline Social Buttons & 24 & $60 \%$ & & \\
\hline Social Share & 12 & $30 \%$ & & \\
\hline Recommend/add to Fav/tag & 4 & $10 \%$ & & \\
\hline total: & 40 & $100 \%$ & 120 & 0,51 \\
\hline \multicolumn{5}{|c|}{$15 \%$} \\
\hline Review & & & 4 & \\
\hline Feedback/comment & 7 & $100 \%$ & & \\
\hline total: & 7 & $100 \%$ & 28 & 0,11 \\
\hline \multicolumn{5}{|c|}{$3 \%$} \\
\hline Participate & & & 5 & \\
\hline Search & 32 & $39 \%$ & & \\
\hline Download & 22 & $26 \%$ & & \\
\hline Ecommerce/Products/Clients & 12 & $15 \%$ & & \\
\hline workshps/trainings & 3 & $4 \%$ & & \\
\hline other events & 10 & $12 \%$ & & \\
\hline Contract research/Service/Projects & 3 & $4 \%$ & & \\
\hline total: & 82 & $100 \%$ & 410 & 1,75 \\
\hline \multicolumn{5}{|c|}{$31 \%$} \\
\hline Collaborate & & & 6 & \\
\hline Collaboration as Expert/volunteer & 2 & $15 \%$ & & \\
\hline Membership/Join & 6 & $47 \%$ & & \\
\hline Donate /support & 5 & $38 \%$ & & \\
\hline total: & 13 & $100 \%$ & 78 & 0,33 \\
\hline \multicolumn{5}{|c|}{$5 \%$} \\
\hline total: & 265 & & & 1,13 \\
\hline
\end{tabular}

Fuente: Elaboración propia.

Como podemos observar, la conexión y la participación son las dimensiones más aplicadas, mientras que la presentación y el intercambio son las segundas. La colaboración y la revisión tienen aquellas con escasa aplicación entre estas organizaciones. Por lo que respecta a los principales factores que determinan las dimensiones, los datos muestran que los centros de medios o las salas de prensa virtuales son el factor determinante para una presentación, el correo electrónico en el caso de la conexión, los botones de los Social Media para compartir y buscar en el caso de la participación. El factor de mayor importancia para determinar la dimensión de la colaboración es la adhesión. El nivel medio de interactividad se registra en 
el nivel medio-bajo, siendo la dimensión de la participación la más interactiva de todas ellas estando por encima de la media $(1,75)$, aunque la búsqueda como una herramienta más pasiva de participación es la más frecuente. Las dimensiones con menor nivel de interactividad son las revisiones y las presentaciones de la organización y su contenido. A pesar de que la colaboración es la dimensión más interactiva e importante para el éxito de la estrategia de relaciones públicas es la que tiene el nivel más bajo de aplicación.

\section{Cuadro 2. La interactividad del contenido.}

\begin{tabular}{|c|c|c|}
\hline Type of content on web & $\begin{array}{l}\text { Formats/Freq Use } \\
\%\end{array}$ & $\%$ of all TT \\
\hline \multirow[t]{2}{*}{ Expositive } & texts & $100 \%$ \\
\hline & Photos & $92.5 \%$ \\
\hline total & $24 \%$ & \\
\hline Medium Interactivity Level & 0,37 & \\
\hline \multirow[t]{2}{*}{ Hyper-textual } & Links & $92.5 \%$ \\
\hline & Hashtags & $33.3 \%$ \\
\hline total & $16 \%$ & \\
\hline Medium Interactivity Level & 0,51 & \\
\hline Participative & maps & $72 \%$ \\
\hline total & $9 \%$ & \\
\hline Medium Interactivity Level & 0,43 & \\
\hline Interactive & Videos/Audio & $85 \%$ \\
\hline \multirow[t]{2}{*}{ total } & $10.5 \%$ & \\
\hline & 0,67 & \\
\hline Immersive & Virtual visits & $0 \%$ \\
\hline total & $0 \%$ & \\
\hline Medium Interactivity Level & 0 & \\
\hline total Medium Interactivity & 2 & \\
\hline
\end{tabular}

Fuente: elaboración propia.

El nivel general de interactividad del contenido es de dos en una escala de tres grados, por lo tanto, es relativamente alto, aunque el tipo de contenido expositivo se utiliza con mayor frecuencia. La mayoría de los think tanks analizados utilizan un contenido media rico e interactivo en sus páginas web.

En términos de utilización de herramientas de medios sociales, en un primer momento hemos determinado las organizaciones con presencia en los Social Media. El cuadro general muestra que solo el $64 \%$ de los think tanks de Asia están presentes en los Social Media. La significativa mayoría de la muestra usa Facebook (96\%) y Twitter (76\%) y el 56\% de ellos tiene blog. 
Cuadro 3. Uso de Social Media.

\begin{tabular}{|l|r|r|r|r|r|r|r|r|}
\hline & Twitter & Facebook & Youtube & Linkedin & Blog & Google+ & Other & $\begin{array}{l}\text { Total } \\
\text { número } \\
\text { de TT en } \\
\text { Social } \\
\text { Media(al } \\
\text { menos1 ) }\end{array}$ \\
\hline $\begin{array}{l}\text { Numero } \\
\text { de TT }\end{array}$ & 19 & 24 & 10 & 7 & 14 & 12 & 4 & 25 \\
\hline $\begin{array}{l}\text { \% de TT } \\
\text { en total }\end{array}$ & $\mathbf{7 6 \%}$ & $\mathbf{9 6 \%}$ & $\mathbf{4 0 \%}$ & $\mathbf{2 8 \%}$ & $\mathbf{5 6 \%}$ & & & \\
\hline
\end{tabular}

Fuente: elaboración propia.

Este modelo dominante de la aplicación de las redes sociales está también reflejado en las redes y en las tácticas que pueden ser determinadas por medio del análisis de la frecuencia de uso de determinadas redes sociales:

Cuadro 4. Uso de Social Media y el nivel medio de la interactividad.

\begin{tabular}{|c|c|c|c|}
\hline SN Usage & $\begin{array}{l}\% \text { of all think tanks } \\
\text { present in SM } \\
\mathrm{N}=25\end{array}$ & $\begin{array}{l}\% \text { of Frequency } \\
\text { Use } N=\mathbf{8 6}\end{array}$ & $\begin{array}{l}\text { Medium } \\
\text { Interactivity } \\
(\mathrm{M} / 25) \\
\end{array}$ \\
\hline Twitter & $76 \%$ & $22 \%$ & 0,15 \\
\hline Facebook & $96 \%$ & $28 \%$ & 0,19 \\
\hline Blog & $56 \%$ & $16 \%$ & 0,11 \\
\hline Youtube & $40 \%$ & $12 \%$ & 0,08 \\
\hline Linkedin & $28 \%$ & $8 \%$ & 0,05 \\
\hline Google Plus & $48 \%$ & $14 \%$ & 0,09 \\
\hline totals & $64 \%$ present in SM & $100 \%$ & 0,68 \\
\hline SN tactics & & $\mathrm{N}=63$ & \\
\hline $\begin{array}{l}\text { Facebook/Twitter } \\
\text { Flickr/Pinterest/Instagram/ }\end{array}$ & $25.4 \%$ & $25 \%$ & 0,25 \\
\hline Slideshare & $1.6 \%$ & $1.6 \%$ & 0,01 \\
\hline Blog/Yotube/vimeo & $9.5 \%$ & $10 \%$ & 0,14 \\
\hline Linkedin/google Plus & $7.9 \%$ & $8 \%$ & 0,12 \\
\hline $\begin{array}{l}\text { Fcb/Twitter/Blog/Youtube } \\
\text { Fcb/Twitter/Google Plus/ }\end{array}$ & $23.8 \%$ & $24 \%$ & 0,48 \\
\hline $\begin{array}{l}\text { Linkedin } \\
\text { Fcb/Twitter/Yt/Blog/Google }\end{array}$ & $11.1 \%$ & $11 \%$ & 0,22 \\
\hline Plus/Linkedin & $20.6 \%$ & $21 \%$ & 0,52 \\
\hline totals & & $100 \%$ & 1,76 \\
\hline
\end{tabular}

Fuente: elaboración propia. 
La táctica más frecuente es Facebook, la segunda es Twitter y, luego, Google Plus. La red Linkedin es utilizada solo en el $8 \%$. En términos del nivel de interactividad, el nivel en todas esas redes es más bien bajo. La elección de las tácticas demuestra un nivel bastante alto de interacción, por encima de la media. La táctica más popular es el uso de la combinación de Twitter y Facebook (25\%) y Facebook/Twitter/Blog y Youtube (24\%) por igual. La última táctica tiene el mayor rendimiento en términos de interactividad, aunque de nuevo el nivel general es más bien bajo.

La estrategia más popular entre los think tanks investigados es la dirigida a la visibilidad y a la magnitud que se basa principalmente en el uso de Twitter y Facebook. En general, esas estrategias resultan adoptadas en su mayoría por su potencial de influencia en el nivel táctico y operativo. Sin embargo, podrían no experimentar el mismo nivel de interactividad con el público. En cuanto a la adopción de redes más especializadas, podemos observar que la gestión de contenido avanzado, la influencia local y la exposición son las que tienen niveles más altos de aplicación. No obstante, la visibilidad y el enfoque de magnitud, así como las networkings profesionales y académicas, parecen ser estrategias elegidas principalmente con propósitos de influencia (especialmente la basada en Twitter y Facebook, así como están teniendo un nivel medio de interactividad por encima de la media.

Midiendo el nivel de innovación, hemos descubierto que en términos generales el nivel es bajo o muy bajo y los think tanks se unieron a las redes sociales en la ola de creciente popularidad de Facebook y Twitter.

De forma parecida, la capacidad de crear una comunidad significativa, aunque forma parte de su estrategia principal, se coloca en niveles medios o incluso niveles aún más bajos,

El mayor número de organizaciones muestran comunidades más bien pequeñas: entre 5012000 fans o seguidores, aunque las comunidades de Facebook de los think tanks de Asia tienden a ser más grandes.

El nivel de interactividad del contenido utilizado como vehículo para conectar e interactuar con estos públicos en Facebook se mantiene en un nivel medio. Sin embargo, los datos muestran que, en lo que respecta a la estrategia de contenido, la utilización más frecuente es más bien modesta en todos los tipos de contenido y posts en Facebook, situada principalmente en un nivel de hasta el $25 \%$, de todo el contenido publicado con un mayor enfoque en los estados (status) y los vídeos como, en menor medida, las publicaciones.

Los think tanks de la región de Asia y el Pacífico presentes en Facebook están teniendo un rendimiento de interactividad en un nivel medio. Los textos son usados por todos ellos, mientras que las fotos y los enlaces lo son por la vasta mayoría. Los think tanks que usan fotografías obtienen los niveles más altos de interactividad. 


\section{Cuadro 5. El nivel del uso de diferentes formatos del contenido.}

\begin{tabular}{|c|c|c|c|c|c|c|c|}
\hline \multicolumn{8}{|l|}{ Post type } \\
\hline Facebook & yes & no & $\%$ yes & VA & Points Awarded & Mean & $\begin{array}{c}\text { Medium } \\
\text { Interactivity }\end{array}$ \\
\hline Video & 7 & 17 & $29 \%$ & 5 & 35 & 7 & 0,28 \\
\hline Photo & 20 & 4 & $83 \%$ & 4 & 80 & 16 & 0,64 \\
\hline hashtags & 5 & 19 & $21 \%$ & 3 & 15 & 5 & 0,2 \\
\hline Links & 19 & 5 & $79 \%$ & 2 & 38 & 7,6 & 0,304 \\
\hline text & 24 & 0 & $100 \%$ & 1 & 24 & 4,8 & 0,192 \\
\hline totals & & & & & 192 & 38,4 & 1,536 \\
\hline
\end{tabular}

Fuente: elaboración propia.

En el caso de Twitter, podemos observar que el uso común de instrumentos interactivos tales como menciones, enlaces y etiquetas para conectar con los diferentes stakeholders es más bien bajo, ya que el uso está muy por debajo de la media (al menos uno de ellos por cada tweet) y el nivel general de interactividad está, por tanto, por debajo de la media.

Para terminar, hemos evaluado el rendimiento general en las dos redes sociales en sus diversas dimensiones.

El nivel de interactividad de Facebook está ligeramente por debajo del nivel medio. Los think tanks en general rinden mejor en términos de construcción de comunidades y contenido y en la dimensión de la actividad contada con la actividad diaria y el tipo de contenido. La interactividad del contenido publicado en Facebook se mantiene también en el nivel medio. La gestión diaria de la fanpage en términos de frecuencia de las publicaciones, sinceridad, uso de herramientas hipertextuales, interacciones con los posts y compromiso de la audiencia se registra en niveles bastante bajos. Teniendo en cuenta que la estrategia centrada en la visibilidad es la elección principal de los think tanks de Asia, ellos rinden en niveles muy bajos en relación con el tráfico y la viralidad que logran obtener

En definitiva, los think tanks de Asia presentes en Twitter están también rindiendo en general en niveles bajos de interactividad, grado de respuesta y disposición a la conversación. La capacidad para construir la comunidad en Twitter es similar a Facebook, sin embargo, el nivel general de interactividad es mucho más bajo. El uso de herramientas hipertextuales es también bajo (aunque superior que en Facebook), al igual que el nivel de actividad. En cuanto a Twitter, es visible que el impacto es más significativo que la interactividad y está ligeramente por encima del nivel medio.

\section{Discusión}

En definitiva, según los resultados analizados, al alcanzar los objetivos planteados, se demuestran en general los valores medios y bajos de interactividad en las páginas web y a través de Social Media, así como la declarada orientación hacia la comunidad y en menor medida hacia los ciudadanos. En su conjunto reflejan la lenta re-orientación hacia estrategias bidireccionales y tácticas de comunidad e influencia en el entorno de la comunicación política 2.0. 
Por otro lado, los insuficientes niveles de interactividad y el mayor peso adjudicado a las estrategias de visibilidad y publicity indican que el modelo común es el modelo bidireccional asimétrico propio de la comunicación organizacional orientada a la difusión de contenido autopromocional hacia los stakeholders, donde los Social Media juegan un papel menos efectivo que los medios de comunicación tradicionales.

Este modelo es bidireccional parcialmente, puesto que la mayoría de los think tanks apuesta por un amplio abanico de soluciones de Social Media (con variedad de grados de realización y gestión), como la estrategia de comunicación de las relaciones públicas políticas 2.0 y simultáneamente integra a las herramientas interactivas. Sin embargo, la realización de esta estrategia se basa en la difusión de los contenidos informativos y autopromocionales hacia los stakeholders claves, con motivo de dar la mayor visibilidad y publicity en las cuestiones políticas de su especialidad o más ampliamente de la actualidad. De este modo, el enfoque principal es la competencia más efectiva y eficaz en el saturado mercado de las ideas.

En este sentido, aunque el uso de los Social Media parece bien asentado y suficientemente extenso, confirmando parcialmente nuestra primera hipótesis, se puede afirmar que cuanto a la diversidad de aplicación de diferentes sitios de Social Media la hipótesis no se confirma ya que las redes más utilizadas se limitan a Facebook y Twitter, principalmente. En cuanto a la segunda hipótesis, dado el carácter autopromocional enfocado en publicity y visibilidad, los think tanks analizados demuestran los niveles demasiado bajos en cuanto a la influencia e involucración con sus stakeholders. Asimismo, la segunda hipótesis no se ha podido confirmar. Todos los indicadores analizados confirman este patrón. El nivel general de interacción y compromiso en todas las redes sociales y en todos los casos es muy bajo y, en los casos de rendimiento más exitoso, es considerable. Esto indica algunos problemas en la creación de la comunidad y la gestión del contenido, así como en el enfoque profesional en la estrategia de relaciones con los stakeholders. Los indicadores incluso más bajos de influencia e impacto confirman la imposibilidad de aplicar correctamente el contenido a las tácticas de contenido e impacto por medio de conversaciones significantes con públicos más amplios. Esto demuestra que la exposición y creación de la comunidad, pero sin consolidación no parece una táctica suficiente hoy en día en las redes sociales. Aunque la interactividad y el compromiso en el caso de algunos think tanks más exitosos parecen tener un nivel aceptable, el impacto o la influencia de los think tanks en cuestión es en general bajo. En cambio, en línea con el enfoque principal de la estrategia de visibilidad, la exposición y el alcance muestran valores más altos que los indicadores de popularidad y relevancia. Esto significa que, aunque la competencia a por una mayor visibilidad en el saturado "mercado de ideas" parece ser el objetivo principal de los think tanks que persiguen convertirse en los actores políticos de referencia debido a sus estrategias de comunicación política 2.0, el nivel general alcanzada no es suficiente, puesto que estos think tanks no se convierten en las fuentes relevantes de información en redes sociales, aún con excepciones.

Teniendo en cuenta estos resultados, el uso limitado de Social Media por los think tanks asiáticos confirma las tendencias observadas en el caso de los think tanks de Europa del Este (Castillo, Almansa y Smolak-Lozano, 2015). Su enfoque mediatico confirma la importancia de uso de los medios de comunicación en sus estrategias (Xifra, 2008), sin embargo, no consiguen ejercer este planteamiento estratégico de los medios de comunicación para influencia política 
e interacción con los públicos de políticas públicas (Abelson, 2006), especialmente en cuanto a Social Media (Selee, 2013 y Mendizabal, 2014).

\section{Conclusiones}

Se puede concluir que las estrategias de comunicación política 2.0 en los Social Media realizadas por los think tanks analizados sirven en primer lugar para la autopromoción y difusión del conocimiento u opinión creados in-house como parte del debate público actual y con motivo de influenciar a la opinión pública (sobre todo a los medios de comunicación, los políticos y los expertos); así como para relacionarse con estos stakeholders y de este modo crear un puente entre el conocimiento y las políticas públicas a través de una amplia difusión del contenido afín con el área de especialidad de cada think tank y sobre todo, con la actualidad. Los social Media definitivamente, según las declaraciones, presentan las ventajas de la difusión de contenido y promoción, incluso de la comunidad, sin embargo, pierden su importancia en las estrategias de relaciones públicas en cuanto a su percibida efectividad en comparación con los medios tradicionales como prensa, radio o televisión. La implementación de las relaciones públicas políticas 2.0 presenta mayores desafíos en cuanto al uso de las herramientas interactivas y adaptación al entorno 2.0 junto con las limitaciones en conocimientos tecnológicos. A pesar de la declarada orientación hacia la comunidad, los niveles de interactividad son medios-bajos y las herramientas interactivas 2.0 dentro de las estrategias de relaciones públicas 2.0 se presentan como un reto, añadiendo a esto la abundancia de contenido autopromocional e informativo, se puede concluir, en base a los valores presentados, que su orientación en cuanto a las relaciones públicas políticas 2.0 es en la mayoría de los casos unidireccional y no bidireccional, sin alcanzar valores altos de influencia y advocacía o sin habilidad de construir una comunidad interconectada fuertemente.

Al cumplir nuestros objetivos de análisis, podemos aproximarnos a la conclusión de que, aunque la orientación hacia la bidireccionalidad está cada vez más patente entre las organizaciones analizadas, el modo de su implementación radica en soluciones unidireccionales y relacionadas con el objetivo principal de acortar la distancia entre los conocimientos y la esfera de las políticas públicas, siendo este el objetivo tradicional de los think tanks globales, que anteriormente realizaban mediante técnicas propias de los medios de comunicación masivos offline.

\section{Referencias}

- Abelson D. (2001). Do The Think tanks Matter? Asessing The Impact Of Public Policy Intitutes. Montreal: McGill-Queen's University Press.

- Abelson, D. (2006). A Capitol Idea. Think tanks And US Foreing Policy. Montreal: McGiII-Queen's University Press.

- Auger, G. (2013). Fostering Democracy Through Social Media: Evaluating Diametrically Opposed Non Profit Advocacy Organizations' Use Of Facebook, Twitter and Youtube, Public Relations Review, 39: 369-376.

- Capriotti P., Castillo Esparcia A., Carretón C. (2016). Testing The Level Of Interactivity Of Institutional Websites: From Museum 1.0 To Muesums 2.0, International Journal of Information Management, 36: 97-104

- $\quad$ Castells, M. (1996). The Rise of Network Society. Oxford, UK: Blackwell Publishers Ltd.

- Castells, M. (2009): Comunicación y Poder. Alianza Editorial, Madrid

- Castillo-Esparcia A. (2009). "Relaciones Públicas Y "Think tanks" En América Latina. Es- 
tudio Sobre Su Implantación Y Acción". Razón Y Palabra: "Relaciones Públicas", Número 70: 1-22.

- $\quad$ Castillo-Esparcia A. , Smolak-Lozano E. (2012). " The Marketing of Ideas of Think tanks as the Communication Strategy on the Political Arena", Zeszyty Naukowe Uniwersytetu Szczecińskiego. Problemy Zarządzania, Finansów i Marketingu, № 23: 496-510.

- Castillo-Esparcia, Almansa-Martínez, A.;Smolak-Lozano, E. (2015): East European Think tanks In Social Media - Towards The Model Of Evaluation Of Effective Communication/ PR Strategies: Case Study Analysis, Catalan Journal Of Communication \& Cultural Studies, Vol. 7, № 2 (Ejemplar dedicado a: Public Relations: Communication, engagement and dialogue), Editor Intellect. Pág. 231-250

- Coombs, T.W., Holladay, S.J. (2015). Public Relationships- Relationships Identity In Research: Enlightenment Or Illusion, Public Relations Review, 41: 689-695.

- del Rey Morató, J. (1996). Democracia y posmodernidadteoría general de la información y comunicación política. Madrid: Universidad y Editorial Complutense.

- del Rey Morató, J. (2007). Comunicación politica, internet y campañas electorales. De la teledemocracia a la ciberdemocracia. Madrid: Tecnos.

- $\quad$ Flores Quintero, G., Arribas Urrutia A. (2008). Incursiones a la complejidad. Comunicación política y sociedad. México: Plaza y Valdés S.A.

- Gershon R.A. (2017). Digital Media and Innovation. Management and Design. Strategies in Communication. London: Sage.

- Herrero, J.C. (2009). Manual de Teoría de la Información y de la Comunicación. Madrid: Editorial Universitas.

- Kuntz F. (2013). "Communications and Impact Metrics for Think tanks", presentation made at the Conference: "Think tanks - Facing the Changing World," hosted by The Chinese Academy of Social Sciences in Beijing, June 17-18, 2013. Recueprado de: https://www.cigionline. org/blogs/tank-treads/communications-and-impact-metrics-think-tanks

- Lalueza, F., Girona R. (2016). "The Impact Of Think tanks On Mass Media Discourse Regarding The Economic Crisis In Spain", Public Relations Review, 42: 271-278.

- Leiner, D., \& Quiring, O. (2008). What interactivity means to the user: essential insights into and a scale for perceived interactivity. Journal Of Computer-Mediated Communication, 14(1), 127-155.

- Mark Verheyden, P. J. (2017): A Theoretical Framework for the Analysis of Messages on Enterprise Social Media, Revista Internacional de Relaciones Públicas, Vol. 7, Núm. 13: 5-22

- McGann, J. G., Johnson E. C. (2005). Comparative Think tanks, Politics and Public Policy. Cheltenham: Edward Elgar Publishing.

- McGann, J.G. (2015). 2014 Global Go To Think tanks Index Report. Philadelphia: University of Pennsylvania. Fuente: http://repository.upenn.edu/think_tanks /8

- Medvetz, T. (2008). Think tanks as an Emergent Field. Lugar no dispoible: The Social Science Research Council.

- Mendizabal E. (2012). Complex Ideas. Translating research into practical social and policy changes. USA: edited by On Think tanks. Licencia Creative Commons 3.0

- Mendizabal E. (2014), " Do Not Dumb Down You Audience -Or Rather, How Should Think tanks Affect Policy Chance?" Publicado en: https://onthinktanks.org/articles/do-notdumb-down-you-audience-or-rather-how-should-think-tanks-affect-policy-chance/ Acceso 12 de Junio de 2016

- Montobbio M. (2013), "Rasgos y razones del desencanto de la democracia en América 
Latina", Foreign Affairs Latinoamérica, vol. 13, n 1, enero.

- $\quad$ Painter D.L. (2015). Online political public relations and trust: Source and interactivity effects in the 2012 U.S. presidential campaign. Public Relations Review. Vol.41/Issue 5. P.801808.

- Ponti Aragón E., Domingo D. (2014). Developing Public Relations 2.0: Practitioners'Perceptions On The Implementation Of Interactive Communication Strategies, Public Relations Review, 40: 559-561.

- Rodriguez Davara J. (no disponible): Estrategias de comunicación en marketing, Dossat, Madrid

- Seitel, F. P. (2002). Teoría y Práctica de las Relaciones Públicas 2.0. Madrid: Pearson Prentice Hall.

- Selee, A. (2013). What Should Think tanks Do? A Strategic Guide to Policy Impact. Stanford: Stanford Briefs.

- Stone, D. (1996). Capturing The Political Imagination: Think tanks and the Policy Process. London: Frank Cass.

- Valentini, C. (2015). Is Using Social Media Good For The Public Relations Profession? A Critical Reflection, Public Relations Review, 41: 170-177.

- Van Dijck, J. (2013). The culture of connectivity. In A critical history of social media. Oxford, UK: Oxford University Press.

- Van Ruler, B. (2015). Agile Public Relations Planning: The Reflective Communication Scrum, Public Relations Review, 41: 187-194.

- Xifra, J. (2003). Teoria y estructura de las relaciones públicas. Madrid: McGraw-Hill.

- Xifra, J. (2008). Los think tanks. Barcelona: Editorial UOC.

- Xifra, J., Ponsa F. (2009). El marketing de las ideas. Los think tanks en España y en el mundo. Barcelona: Editorial UOC.

\section{NOTAS:}

(1) La presentación en el congreso: Think tanks Facing the Changing World, organizada por The Chinese Academy of Social Sciences en Beijing, China, June 17-18, 2013.

(2) La presentación completa disponible en: https://onthinktanks.org/articles/communication-options-for-think-tanks-channels-and-tools/ 


\section{CURRICULUM VITAE}

\section{Antonio Castillo Esparcia}

Catedrático de Universidad. Docente en la Universidad de Málaga. Especialista Relacio-nes Públicas, Comunicación Política e Investigación en Comunicación. Autor de más de 150 publicaciones entre artículos en revistas, libros y capítulos de libros en editoriales nacionales y extranjeras. Director del Proyecto de Investigación Lobby y Comunicación, financiado por el Programa Estatal de I+D+i (CSO2016-79357-R). Director del Grupo de Investigación Las Relaciones Públicas en la pequeña y Mediana Empresa.

\section{Emilia Smolak Lozano}

Doctora en Comunicación por Universidad de Málaga y Profesora de Relaciones Publicas y Publicidad en Departamento de Comunicación Audiovisual y Publicidad en la misma. Licenciada en Sociología (Universidad Jaguellónica) y Estudios Europeos (Universidad de Exeter) es autora de casi 30 artículos sobre la temática de relaciones públicas, comunicación política y Social Media, así como ponente de más de 30 congresos en las ciencias de la comunicación. En 2017, ha sido co-autora del libro sobre la comunicación política 2.0 de los think tanks y lobby. 